\title{
Interphalangeal Thumb Joint
}

National Cancer Institute

\section{Source}

National Cancer Institute. Interphalangeal Thumb Joint. NCI Thesaurus. Code C102306.

A ginglymoid (hinge) synovial joint within the thumb connecting the proximal and distal phalanges. 